\title{
Novel Planar Capacitive Interdigital Multifinger (IDCM) Sensor for the Detection of the Ageing of Electrical Transformer Oil
}

\author{
R. G. M. Oliveira ${ }^{* 1}$, J. E. V. de Morais ${ }^{1}$, V. L. Bessa ${ }^{1,2}$, M. C. S. Freitas ${ }^{3}$, V. C. Martins ${ }^{1}$, M. A. S. Silva ${ }^{1}$, H. D. \\ de Andrade ${ }^{4}$, I. S. Queiroz Júnior ${ }^{4}$ and A. S. B. Sombra ${ }^{1,4}$ \\ ${ }^{1}$ Telecommunication and Materials Science and Engineering of Laboratory (LOCEM), Physics Department, Federal University of \\ Cear(UFC), Campus PICI, Fortaleza, Brazil \\ ${ }^{2}$ Teleinformatics Engineering, Federal University of Cear, Pici Campus, Fortaleza, Brazil \\ ${ }^{3}$ Federal Institute of Cear, Campus Fortaleza/IFCE, Fortaleza, Brazil \\ ${ }^{4}$ Federal University of Semiarid Region, UFERSA, Mossoro, Brazil \\ E-mail: ronaldomaia@fisica.ufc.br
}

\begin{abstract}
This work shows a planar interdigital sensor for rapid measurement of oils. The kit is characterised by the impedance meter, a Raspberry Pi3 and a sample port where the planar capacitive interdigital multifinger (IDCM) sensor is stored. For validation of this IDCM sensor, the data was compared with measurements performed by impedance spectroscopy (IS). The measurements show that the measured values of the new and used oils are very close, validating the IDCM sensor for fast oil measurements of oils in transformers and other devices.

Keywords: Planar Interdigital Capacitor, Permittivity, Transformer Oil
\end{abstract}

\section{Introduction}

Electricity industries currently require high safety precautions, especially in the maintenance of transformers, which demand time and high cost. In this context, it is important that a periodic analysis of the state of conservation of the oil contained in the transformer be held. For these analyses, refined techniques such as chromatography and UV-Vis spectroscopy are utilised that are expensive and time consuming. Moreover, new techniques are being developed for in-situ analysis with the same precision as other techniques.

In the literature some works with interdigital sensors, called planar capacitive interdigital multifinger (IDCM), present many applications in the most diverse areas such as chemical, biological, microelectromechanical, telecommunications, and piezoacoustic systems ${ }^{[1-6]}$. New types of interdigital sensors having the most diverse configurations, with different numbers of electrodes, are being studied for various types of projects ${ }^{[7-10]}$. The manufacturing process involves different types of substrates amongst which, FR4 can be cited for the cost-effectiveness and manufacturing quality of the circuit ${ }^{[1]}$. The planar interdigital circuit is a parallel plate capacitor ${ }^{[12]}$ in which the electric field lines, generated by the sensors, pass through the material to be studied and directly influence the sensor impedance. The sensor follows the principle of a capacitor in which the reactive capacitance of the properties of the system, surrounding the sensor, can be measured and calculated $^{[8]}$. The permittivity of a material is the result between the relative permittivity $\left(\varepsilon_{r}\right)$ and the permittivity in vacuum $\left(\varepsilon_{o}\right)$, shown in Equation 1.

$$
\varepsilon=\varepsilon_{r} \varepsilon_{o}
$$

The relative permittivity of an insulating material, shown in Equation 2, can be calculated by the relationship between the capacitance of a capacitor $(C)$, where the area between the electrodes is filled with an insulating material, and the capacitance in a vacuum $\left(C_{o}\right)$.

$$
\varepsilon=\frac{c}{c_{0}}
$$


According to Rahman et al. ${ }^{[7]}$, the term interdigital, in the acronym IDCM, refers to a digit-like, or finger-like, periodic pattern of parallel in-plane electrodes, used to build up the capacitance associated with the electric fields that penetrate into a sample material ${ }^{[2]}$, so that a voltage source can be applied between the positive terminal and the negative terminal ${ }^{[8]}$ and is a technique to measure the permittivity \&r of fluids through the capacitance in systems with multiple capacitors ${ }^{[3,7]}$.

The characteristics of the studied material can be determined by the change in capacitance of the IDCM, filled with the fluids (oils for example) studied and is estimated by Equation $3^{[13]}$, where $\mathrm{n}$ is the number of parallel plates, A, the area of one side of each plate and $d$ is the thickness of the dielectric ${ }^{[3]}$.

$$
c=\frac{\varepsilon_{r} \varepsilon_{0}(n-1) A}{d}
$$

This work shows the fabrication and use of a 7-finger, where each pair of fingers corresponds to a flat capacitor of the IDCM sensor ${ }^{[14,15]}$ for fast measurements of the transformer oils and the lifting of a calibration curve referring to the quality of these oils. Each oil measurement is performed in a maximum of $2 \mathrm{~min}$ and the result is sent to a company monitoring central, allowing in a few minutes, to suspend the use of the transformer, if the oil shows undesirable variations. For this measurement, a kit was developed that consists of a capacitance meter and a Raspberry PI3 for communication and transmission of the collected data. A study carried out by the software Comsol was extremely important, since the IDCM was built based on simulations that verified the effectiveness of the project, as shown in the results of this work.

\section{Experimental Setup}

The flat interdigital capacitor with parallel plates was built with copper sheets of thickness $0.2 \mathrm{~mm}$ on a substrate of the FR4 epoxy ( $\varepsilon=4.4$ and thickness $1.4 \mathrm{~mm}$ ). To identify the best thickness, length and distance between the fingers of the IDCM, the software COMSOL Multiphysics was used ${ }^{\mathbb{Q}[8]}$, resulting in the dimensions shown in Figure 1(a).
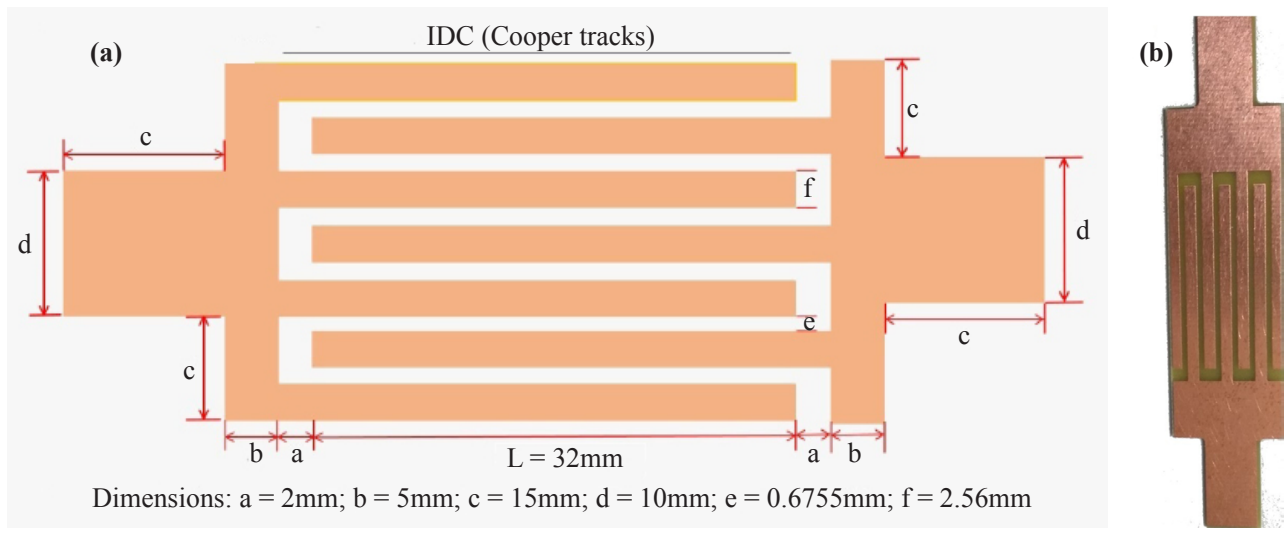

Figure 1. Interdigital capacitor. (a) Projected dimensions of IDCM, (b) IDCM produced

Figure 1(b), presents the designed and manufactured IDCM, which will be used for the measurements of the oils. To control the amount of oil in all measurements, a support for the IDCM was designed and built and this model is shown in Figure 2.

After the capacitance measurement, the numerical value is sent to the developed software and installed in the raspberry PI3, where, through the measured capacitance, the relative permittivity was calculated thus estimating the relative permittivity value of all the measured oils. These were compared with the oils that were measured by impedance spectroscopy (IS) on a Solartron 1260 model in the frequency range of $1 \mathrm{~Hz}$ to $1 \mathrm{MHz}$. 
Table 1. Oils analysed

\begin{tabular}{cccccccccc}
\hline Oil & $\boldsymbol{\varepsilon}_{\mathbf{r}}$ & Oil & $\boldsymbol{\varepsilon}_{\mathrm{r}}$ & Oil & $\boldsymbol{\varepsilon}_{\mathrm{r}}$ & Oil & $\boldsymbol{\varepsilon}_{\mathrm{r}}$ & Oil & $\boldsymbol{\varepsilon}_{\mathrm{r}}$ \\
\hline New (IDCM) & 2.401 & & & & & & & & \\
101 & 2.442 & 111 & 2.411 & 121 & 2.383 & 131 & 2.379 & 141 & 2.445 \\
102 & 2.371 & 112 & 2.371 & 122 & 2.399 & 132 & 2.304 & 142 & 2.409 \\
103 & 2.388 & 113 & 2.353 & 123 & 2.369 & 133 & 2.363 & 143 & 2.418 \\
104 & 2.387 & 114 & 2.323 & 124 & 2.331 & 134 & 2.432 & 144 & 2.409 \\
105 & 2.372 & 115 & 2.427 & 125 & 2.144 & 135 & 2.445 & 145 & 2.395 \\
106 & 2.442 & 116 & 2.358 & 126 & 2.285 & 136 & 2.442 & 146 & 2.441 \\
107 & 2.420 & 117 & 2.420 & 127 & 2.414 & 137 & 2.391 & 147 & 2.451 \\
108 & 2.424 & 118 & 2.398 & 128 & 2.361 & 138 & 2.372 & 148 & 2.421 \\
109 & 2.467 & 119 & 2.372 & 129 & 2.377 & 139 & 2.378 & 149 & 2.407 \\
110 & 2.353 & 120 & 2.462 & 130 & 2.397 & 140 & 2.446 & 150 & 2.374 \\
\hline
\end{tabular}

The support for the IDCM guarantees the amount of oil to be measured and can be used from $1 \mathrm{ml}$ up to $10 \mathrm{ml}$. The oils volume control is made through a Pasteur pipette. In the measurements made with $\mathrm{p}$ to $10 \mathrm{ml}$. The oils volume control is made through a Pasteur pipette. In the measurements made with the new and used oils, an amount of $2 \mathrm{ml}$ of oil was used to calculate the capacitance and permittivity. After the oil is deposited on the holder, the reading is performed through a capacitance meter, Minipa MX -1050, as shown in Figure 3, where the frequency ranges from $100 \mathrm{~Hz}$ to $100 \mathrm{kHz}$.
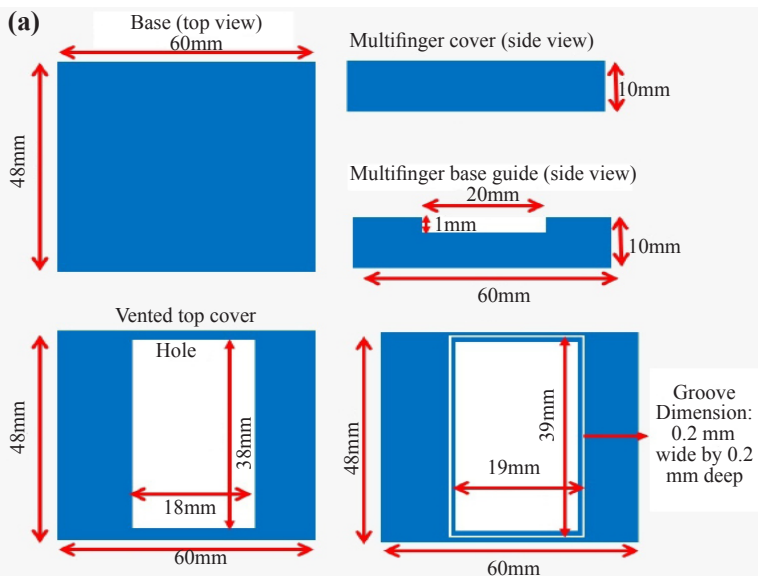

(b)

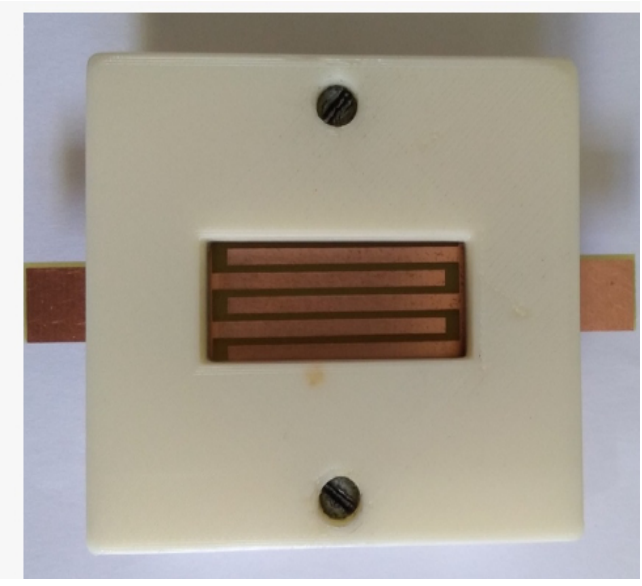

Figure 2. Interdigital capacitor. (a) Projected dimensions for IDCM, (b) Support for the oil control over IDCM

The multifinger kit makes quick oil measurements, so do does not take moisture into account. The measurements only certify whether the oils are working within a pattern or not. For more accuracy measurements involving other parameters that should be studied other techniques will be indicated. In this study, it was used as a measurement standard to validate the results obtained in the multifinger, the solartron 1260. In the solartron 1260, the error expressed by measurement is on the order of $10^{-3}$, while in the impedancimeter is on the order of $10^{-1}$, which is an acceptable price for this type of measure, as the Impedance deviation is given by the Equation 4, where D is the measured percentage deviation, DC is the main parameter of the measured elements, DREF is nominal value, shown on the impedance meter display.

$$
D \%=\frac{D_{c} * D_{R E F}}{D_{R E F}} * 100
$$




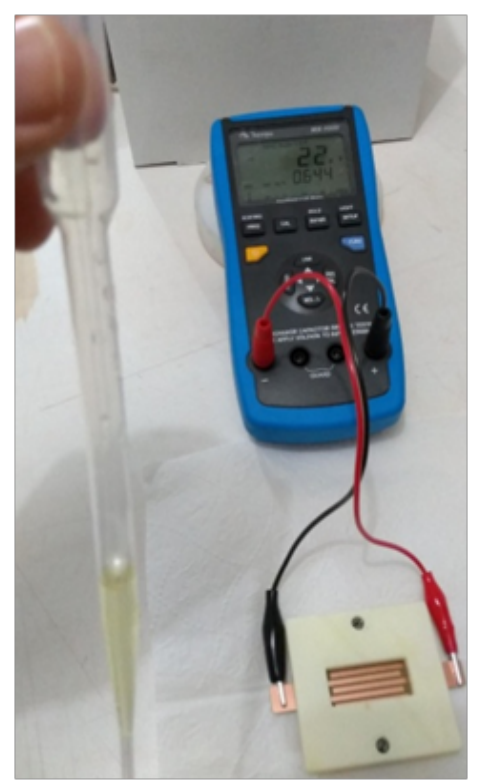

Figure 3. Mounted system for the measurement of oil capacitance

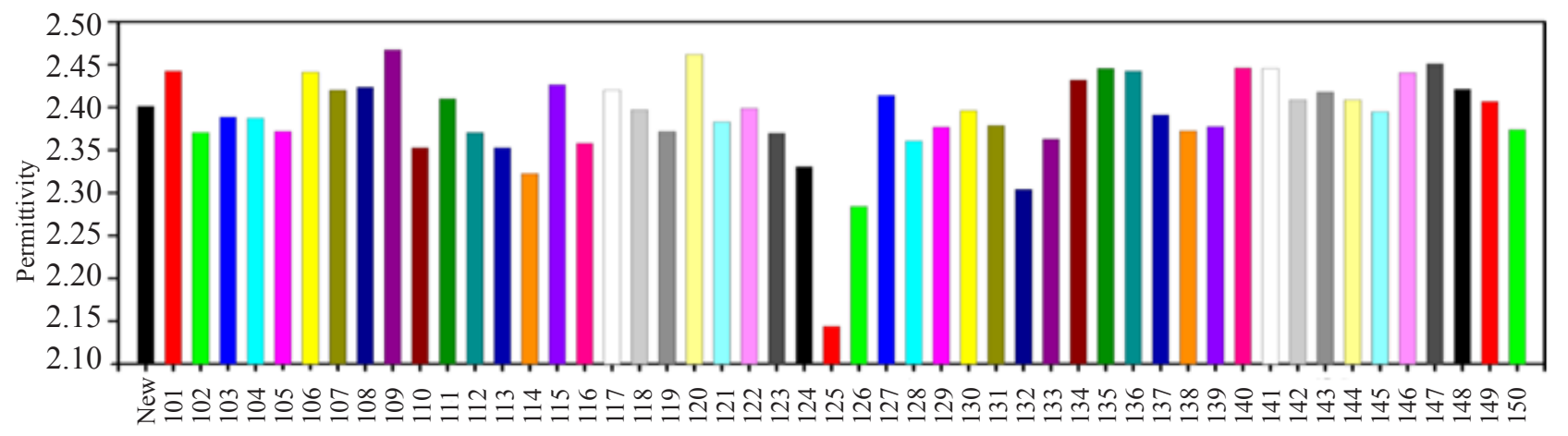

Figure 4. Values of the 51 analysed oil

\section{Experimental Results and Discussions}

For comparing and validating the method, initially water and isopropyl alcohol were measured with the IDCM, where their respective permittivity was 80 and 18 for a frequency of 100KHz. The IDCM measured empty presents a capacitance value of the $10.69 \mathrm{pF}$, at $100 \mathrm{KHz}$.

Knowing the capacitance when there is only air in the sample port, the capacitance of the studied oil is measured by calculating the oil permittivity through Equation 2. From the literature, it is known that the permittivity of new transformer oils is around $\varepsilon \approx 2.40^{[16-18]}$, with oils that fall outside this value being oils that have been used or damaged. The oils analysed in this work vary from new oil to others in different states of conservation and use. The dielectric measurements of theses samples, performed through the IDCM, are shown in Table 1.

As can be seen in Table 1, the values of the new oils measured by the IDCM are compatible with oils studied in the literature, where the dielectric constant values are measured according other techniques, showing the efficiency ${ }^{[14,19]}$. Note that the impedance meter measurement is a very fast measurement not taking into account air humidity, the measurement error is negligible since the are compatible with the solatron results and quality of the measurements by the IDCM. Table 1 shows a comparison between the oils measured in the IDCM, and by IS, at a specific frequency of $100 \mathrm{KHz}$, showing the proximity between the values.

The values that are different from the range of values considered normal (new oil) can confirm that in this case the oils may have either lost their chemical properties, or that there was a contamination or a change in the chemical composition of the oil. For example, water with a permittivity around 80 is consider a contaminant for oil. Figure 4 shows 51 oils and it can be seen that the permittivity of all the oils present close values, thus creating a standard to establish a pattern curve for the IDCM, since through this curve a quick measurement of the oils can be made. 
A $2 \mathrm{ml}$ oil sample is placed inside the IDCM and the data obtained must be compared to the system database for the oil quality information to be obtained. A value is quickly presented within the established calibration curve, with the measurement presenting a tolerance factor of $2 \%$, which was established by experimental measurements and by more detailed observations of Figure 4.

Many oils were measured, and their permittivity was calculated for comparison with respect to the new oil. A tolerance factor of $2 \%$ was determined to estimate the physical quality of the oil. If the permittivity of the oil is within one per cent, relative to the permittivity of the new oil, this means that the oil still retains its properties and can be used for some time. If the difference in permittivity of the new oils is between one percent and two percent, this means that attention must be paid to the oil and its properties analysed for its suitability for use. If the difference is greater than two percent, this means that the oil should be changed immediately, where we can highlight that oil 125 needs to be changed immediately, as it presents a $10.7 \%$ percentage change in relation to new oil, representing a risk to the transformer. Of the analysed samples, oil 126 presents degree of impurities around 4.83\%, also representing a risk of use. The tolerance value was established by the measurement of many oils: new, burned, and used oils.

The kit consisting of the impedance meter, sample holder and the IDCM, has been validated and has become another effective method for the rapid measurement of transformer oils, and other types of uses in the commercial area, since it is easy to handle, has low cost and is very efficient. The studies showed that through the capacitance, the permittivity is obtained, which represents a set of properties of the oil, and from this value it is possible to classify the oil as NEW oil (in perfect state), deserves ATTENTION (make a new check in half of the forecast time, that is, 6 months reviewing in 3 months) and DANGER (a more detailed analysis of the oil needs to be done).

\section{Conclusion}

In view of the studies carried out, it can be concluded that the IDCM presents itself as an optimum option for verifying the quality of the oils used in transformers, being able to estimate their quality quickly and efficiently. The validation of the measurements of the IDCM were carried out comparing the measurements made by impedance spectroscopy and the values were found to be comparable. Therefore, the IDCM in conjunction with an impedance meter, a Raspberry Pi3 and a sample port, where the IDCM sensor is placed, are a kit for rapid measurement of fully functioning oils and can be a powerful tool in the analysis of transformer oils in hydroelectric plants and other industrial devices.

\section{Acknowledgments}

The authors are grateful to CNPq (402045/2013-0), the US Air Force Office of Scientific Research (AFOSR) (FA9550-16-1-0127), CNPq (Process: 402561/2007-4, Edital MCT/CNPq n ${ }^{\circ} 10 / 2007$ ) for providing financial support and Foundation for Research Support of the State of Mato Grosso (FAPEMAT). Acre Electricity Company (ENERGISA).

On behalf of all authors, the corresponding author states that there is no conflict of interest.

\section{References}

[1] Abu-Abed A S, Lindquist R G. Capacitive interdigital sensor with inhomogeneous nematic liquid crystal film. Progress In Electromagnetics Research B. 2008; 7: 75-87. Available from: doi:10.2528/PIERB08022901.

[2] Mamishev A V, Sundara-Rajan K, Du F Y Y, Zahn M. Interdigital sensors and transducers. Procceedings of the IEEE. 2004; 92(5): 808-845. Available from: doi: 10.1109/JPROC.2004.826603.

[3] Bolton W. Engineering Science. 5th ed. Oxford: Elsevier; 2006.

[4] Li L, Uttamchandani D. A concept of moving dieledtrophoresis electrodes based on microelectromechenical systems (MEMS) actuators. Progress In Electromagnetics Research Letters. 2008; 2: 89-94. Available from: doi: 10.2528/ PIERL0712281.

[5] Ngo T-T, Shirzadfar H, Kourtiche D et al. A Planar Interdigital Sensor for Bio-impedance Measurement: Theoretical analysis, Optimization and Simulation. Journal of Nano-Electronic Physics. 2014; 6(1): 01011-1 - 01011-7. Available from: https://jnep.sumdu.edu.ua/en/component/content/full_article/1182 [Accessed 14th April 2017].

[6] Bosse S, Lehmhus D, Lang W et al. Material-Integrated Intelligent Systems Technology and Applications. Germany: Willey; 2018.

[7] Terzic E, Terzic J, Nagarajah R et al. Capacitive Sensing Technology, in A Neural Network Approach to Fluid Quantity Measurement in Dynamic Environments. London: Springer; 2012.

[8] Rahman M S A, Mukhopadhyay S C, Yu P-L. Novel Planar Interdigital Sensors. Novel Sensors for Food Inspection: 
Modelling, Fabrication and Experimentation, Smart Sensors, Measurement and Instrumentation. Switzerland: Springer; 2014. p.11-35.

[9] Chen T, Bowler N. Design of interdigital spiral and concentric capacitive sensors for materials evaluation. 2013; 1155(1593): 1593-1600. Available from: doi: 10.1063/1.4789232.

[10] Ngo T-T, Bourjilat A, Claudel J et al. Design and Realization of a Planar Interdigital Microsensor for Biological Medium Characterization. Next Generation Sensors and Systems. Switzerland, Springer; 2016. p.23-54.

[11] Lee T, Kim C, Sung M et al. Flexural and tensile moduli of fl exible FR4 substrates. Journal Electronic Resource. 2016; 53:70-76. Available from: https://www.tib.eu/en/search/id/elsevier\%3Adoi 10.1016\%252Fj. polymertesting.2016.05 .012/Flexural-and-tensile-moduli-of-flexible-FR4-substrates/?tx_tibsearch_search\%5B searchspace $\% 5 \mathrm{D}=\operatorname{tn}$ [Accessed 25th June 2017].

[12] Samant, H, Jha A K, Ansari M A H et al. Design of CPW fed IDC resonator for non invasive testing of chemical solvents. 2015 IEEE 16th Annual Wireless and Microwave Technology Conference (WAMICON), 13-15 April 2015. USA: IEEE; 2015. p.1-4. Available from: doi:10.1109/WAMICON.2015.7120405.

[13] Terzic E, Terzic J, Nagarajah R et al. A Neural Network Approach to Fluid Quantity Measurement in Dynamic Environments. London: Springer; 2012.

[14] Hu X, Yang W. Planar capacitive sensors - designs and applications. Sensor Review. 2010; 30(1): 24-39. Available from: doi:10.1108/02602281011010772.

[15] Lehmhus D, Bosse S. Material-integrated Intelligent Systems: A Review on State of the Art, Challenges and Trends. Proceedings of 2nd International Electronic Conference on Sensors and Applications, 15-30 November 2015, Switzerland. Basel: MDPI; 2015. p.1-9.

[16] Ten C F, Fernando M A R M, Wang Z D. Dielectric properties measurements of transformer oil, paper and pressboard with the effect of moisture and ageing. 2007 Annual Report - Conference on Electrical Insulation and Dielectric Phenomena. 14-17 October 2007. Canada: IEEE; 2007. p.727-730. Available from: doi:10.1109/ CEIDP.2007.4451521.

[17] Thabet A, Shaaban S, Allam M. Enhancing dielectric constant of transformer oils using multi-nanoparticles technique under thermal conditions. 2016 Eighteenth International Middle East Power Systems Conference (MEPCON), 27-29 December 2016. Egypt: IEEE; 2016. p.220-225. Available from: doi:10.1109/MEPCON.2016.7836894.

[18] Abdi S, Boubakeur A, Haddad A. Influence of thermal ageing on transformer oil properties. 2008 IEEE International Conference on Dielectric Liquids, 30 June-3 July 2008. France: IEEE; 2008. p.1-4. Available from: doi:10.1109/ ICDL.2008.4622466.

[19] Syaifudin A R M, Yunus M A, Mukhopadhyay S C et al. A novel planar interdigital sensor for environmental monitoring. 2009 IEEE Sensors, 25-28 October 2009. New Zealand: IEEE; 2009. p.105-110. Available from: doi: 10.1109/ICSENS.2009.5398227. 